\title{
THE MAP OF THE WITT RING OF A DOMAIN INTO THE WITT RING OF ITS FIELD OF FRACTIONS
}

\author{
THOMAS C. CRAVEN, ALEX ROSENBERG AND ROGER WARE 1
}

ABSTRACT. Let $R$ be an integral domain with field of fractions $K$. This paper studies the kernel of the map $W(R) \rightarrow W(K)$, where $W$ is the Witt ring functor. In case $R$ is regular and noetherian, it is shown that the kernel is a nilideal. The kernel is zero if $R$ is a complete regular local noetherian ring with 2 a unit. Examples are given to show that the regularity assumptions are needed.

1. Introduction and notations. For any commutative ring, let $W(R)$ denote the Witt ring of symmetric nondegenerate bilinear forms over $R$ as defined in [5]. In this note we study the kernel of the homomorphism $W(R) \rightarrow$ $W(K)$ induced by the inclusion of a noetherian integral domain in its field of fractions $K$. By [5, Satz 11.1.1] this kernel is zero if $R$ is Prüfer. In $\$ 2$, we show that the kernel is a nilideal if $R$ is a regular noetherian domain, and that it is even zero if $R$ is a complete noetherian regular local ring with 2 a unit. In $\$ 3$, we give some applications of these results and also provide some examples to show that the regularity hypothesis is needed. We have not been able to determine whether the kernel is zero or not in case $R$ is a regular noetherian local ring with 2 a unit.

We shall consistently use the following notations: For any commutative ring $R$, we write $U(R)$ for the group of units of $R$. If $P$ is a prime ideal of $R$, we write $K(P)$ for the field of fractions of $R / P$, i.e., $K(P) \cong R_{P} / P R_{P}$. If $R$ is a domain we write $K$ for $K(0)$. We shall also write $R^{2}$ for the set of squares in $R$.

By a space $E$ over $R$, we mean a finitely generated projective $R$-module together with a symmetric nondegenerate bilinear form $\Phi$. If $E$ is a space over $R$ then $[E]$ denotes its equivalence class in $W(R)$. We shall write $\left(a_{1}, \cdots, a_{n}\right)$ to denote the space $E$ which is a free $R$-module with basis

Received by the editors June 10, 1974.

AMS (MOS) subject classifications (1970). Primary 15A63; Secondary 13 H05.

1 The second author gratefully acknowledges support from NSF Grant GP-25600, and the third from NSF Grant GP-37781. 
$x_{1}, \cdots, x_{n}$ and form given by $\Phi\left(x_{i}, x_{j}\right)=a_{i} \delta_{i j}$

2. The kernel. We begin with the following proposition whose proof is to some extent inspired by [6, Corollary 2.3].

Proposition 2.1. Let $R$ denote a regular noetherian local ring with maximal ideal M. Then $\operatorname{Ker}(W(R) \rightarrow W(K))$ is contained on $\operatorname{Ker}(W(R) \rightarrow W(R / M))$.

Proof. We use induction on the Krull dimension, $\operatorname{dim} R$, of $R$. If $\operatorname{dim} R=0$, then $R=K$ and $M=0$ so that both kernels are 0 . Now, suppose $\operatorname{dim} R>0$, let $t$ denote a member of a regular system of parameters of $R$ [12, p. 301], and let $P=R t$. Then $\bar{R}=R / P$ is a regular local ring with $\operatorname{dim} \bar{R}=\operatorname{dim} R-1$ [12, Theorem 26, p. 303 and Corollary 2, p. 291], so that $P$ is a prime ideal. Moreover, $R_{P}$ is a regular local ring of dimension one so that $R_{P}$ is a rank one discrete valuation ring [10, 4.7-4.8, pp. 73-76]. Let $E$ be an $R$-space with $\left[E \otimes_{R} K\right]=0$ in $W(K)$. By [5, Satz 11.1.1], $W\left(R_{P}\right) \rightarrow W(K)$ is injective, so that $\left[E \otimes_{R} R_{P}\right]=0$ in $W\left(R_{P}\right)$, which in turn yields $\left[E \otimes_{R} K(P)\right]=0$ in $W(K(P))$. Now $E \otimes_{R} K(P) \cong\left(E \otimes_{R} \bar{R}\right) \otimes_{\bar{R}} K(P)$ and $K(P)$ is the field of fractions of $\bar{R}$. Hence, since the residue class field of $\bar{R}$ is also $R / M$, the induction hypothesis yields $\left[\left(E \otimes_{R} \bar{R}\right) \otimes_{\bar{R}} R / M\right]=$ $\left[E \otimes_{R} R / M\right]=0$ in $W(R / M)$.

A commutative noetherian domain is called regular if for all prime ideals $P$ of $R$, the local rings $R_{P}$ are regular. It is a famous theorem of AuslanderBuchsbaum-Serre [3, Theorem 14, p. 185] that a regular local ring is regular in this sense.

Corollary 2.2. Let $R$ be a noetherian regular domain. Then

$$
\operatorname{Ker}(W(R) \rightarrow W(K))=\bigcap_{P \text { in Spec }(R)} \operatorname{Ker}(W(R) \rightarrow W(K(P))) .
$$

Proof. Since 0 is a prime ideal the right-hand side if clearly contained in the left-hand side. For any prime ideal $P$. of $R$, Proposition 2.1 shows that $\operatorname{Ker}\left(W\left(R_{P}\right) \rightarrow W(K)\right)$ lies in $\operatorname{Ker}\left(W\left(R_{P}\right) \rightarrow W(K(P))\right)$. Hence if $E$ denotes an $R$-space with $\left[E \otimes_{R} K\right]=0$ in $W(K)$, the class $\left[E \otimes_{R} R_{P}\right]$ lies in $\operatorname{Ker}\left(W\left(R_{P}\right) \rightarrow W(K(P))\right)$, i.e., $\left[\left(E \otimes_{R} R_{P}\right) \otimes_{R_{P}} K(P)\right]=\left[E \otimes_{R} K(P)\right]=0$ in $W(K(P))$, so that the left-hand side is contained in the right-hand side.

Corollary 2.3. Let $R$ be a regular noetherian local ring with maximal ideal $M$, such that $1+M \subset R^{2}$. (This bypothesis is fulfilled if $R$ is complete or only henselian.). If 2 is in $U(R)$, then $W(R) \rightarrow W(K)$ is injective.

Proof. By the proof of $[5$, Satz 7.1.11(b)] the mapping $W(R) \rightarrow W(R / M)$ is an injection. 
Remark. Corollary 2.3 was originally proved by one of us [2] assuming 2 in $U(R)$ and $R$ complete. M. Knebusch then pointed out that Corollary 2.3 also followed from [6, Theorem 3.1] as was noted in the Addendum of [2].

Theorem 2.4. Let $R$ be a regular noetherian local ring. Then $\operatorname{Ker}(W(R) \rightarrow W(K))$ is nil, whence by [8, Example 3.11] it is an abelian torsion group.

Proof. Let $E$ be an $R$-space such that $\left[E \otimes_{R} K\right]=0$ in $W(K)$. It is sufficient to show that $[E]$ lies in every minimal prime ideal $\$$, of $W(R)$. By [8, Example 3.11] either $W(R) / \Re \cong Z / 2 Z$ or $\mathbf{Z}$. By the same reference, in the first case, $\$$ consists of all spaces of even rank and since $\left[E \otimes_{R} K\right]=0$ shows that the rank of $E$ is even, $[E]$ lies in $\$$. If $w(R) / \Re \cong \mathbf{Z}$ then by [7, Theorem 4.7], there is a prime ideal $P_{0}$ of $R$ and a nonmaximal prime ideal $\cong$ of $W\left(K\left(P_{0}\right)\right)$ such that $\mathfrak{\beta}$ is the inverse image of $\cong$ with respect to the map $W(R) \rightarrow W\left(K\left(P_{0}\right)\right)$. By Corollary 2.2, we have $\left[E \otimes_{R} K\left(P_{0}\right)\right]=0$ in $W\left(K\left(P_{0}\right)\right)$, so that $\left[E \otimes_{R} K\left(P_{0}\right)\right]$ lies in $\cong$ whence $[E]$ lies in $\Re$.

Corollary 2.5. (a) If $R$ is a regular noetherian local ring then every signature of $R[9$, Definition 2.1] is induced by an ordering of $K$.

(b) If $R$ is a regular domain and for some maximal ideal $M$ of $R$ the field $R / M$ is formally real, then so is $K$.

Proof. The first assertion is immediate from [9, Corollary 2.11]. As for the second, since $R / M$ is formally real, there is a ring surjection $W(R / M) \rightarrow \mathbf{Z}\left[11\right.$, Satz 22]. Composing this with $W\left(R_{M}\right) \rightarrow W(R / M)$ yields a ring surjection $W\left(R_{M}\right) \rightarrow \mathbf{Z}$ which in turn yields a signature on the regular local ring $R_{M}$. By (a), this is induced by an ordering of $K$, so that $K$ is formally real.

Of course, it is easy to prove Corollary 2.5(b) directly: To do this it is enough to assume $R$ is local and use induction on $\operatorname{dim} R$. If $\operatorname{dim} R=1$ then $R$ is a valuation ring. If $K$ is not formally real then by clearing denominators, we get an equation $\sum_{i=1}^{r} x_{i}^{2}=0$, where $x_{i}$ in $R$ are not all zero. Since $R$ is a valuation ring one of the $x_{i}$ 's, say $x_{1}$, will divide all the others so we obtain a relation $-1=\Sigma_{i=2}^{r} y_{i}^{2}$ with $y_{2}, \ldots, y_{r}$ in $R$. Mapping this equation to $R / M$ shows that $R / M$ is not formally real. Now suppose $\operatorname{dim} R=n>1$ and let $P$ be a nonzero principal ideal of $R$. Then $R_{P}$ is a valuation ring with residue class field $K(P)$ so by the above it is enough to show $K(P)$ is formally real. But $R / P$ is a regular local ring of dimension $n-1$ with residue class field $R / M$ and field of fractions $K(P)$, so by the induction asumption, $K(P)$ is formally real. 
In the case that $R$ is a (not necessarily noetherian) valuation ring, the foregoing proof is due to Lang. Note that by localizing we also get a direct proof of Corollary 2.5(b) when $R$ is a Prüfer domain.

Corollary 2.6. Let $R$ be a regular noetherian local ring with 2 in $U(R)$ and suppose that $K$ is pythagorean (i.e., the sum of elements of $K^{2}$ is in $K^{2}$ ), then $W(R) \rightarrow W(K)$ is injective.

Proof. By Theorem 2.4 it is sufficient to prove that $\mathrm{Nil}(W(R))$, the nil radical of $W(R)$, vanishes. Now [9, Theorem 4.18] shows that $\mathrm{Nil}(W(R))$ is generated by all classes $[(1,-b)]$ with $b$ in $U(R) \cap$ \{ sums of elements of $\left.R^{2}\right\}$. Since $K$ is pythagorean, $b=k^{2}$ for some $k$ in $K$. Thus $k$ is integral over $R$. Since $R$ is regular, it is integrally closed, which shows that $k$ is in $U(R)$ so that $[(1,-b)]=0$ in $W(R)$.

In order to obtain a global version of Theorem 2.4 we need the following result of $A$. Dress which appears as Theorem 2.4 in [7]: ${ }^{2}$

Let $R$ denote an arbitrary commutative ring. For any minimal prime ideal $\Re$ of $W(R)$ there exists a maximal ideal $M$ of $R$ and a minimal prime ideal $\cong$ of $W\left(R_{M}\right)$ such that $\Re$ is the inverse image of $\cong$ with regard to the map $W(R) \rightarrow W\left(R_{M}\right)$.

As an immediate consequence we have

Corollary 2.7. Let $R$ be any commutative ring and $E$ any $R$-space such that $\left[E \otimes_{R} R_{M}\right]$ is nilpotent in $W\left(R_{M}\right)$ for all maximal ideals $M$ of $R$. Then $[E]$ is nilpotent in $W(R)$. In particular, the kernel of $W(R) \rightarrow \Pi W\left(R_{M}\right)$, where the product extends over all maximal ideals of $R$, is a nilideal.

Theorem 2.8. Let $R$ be a noetherian regulardomain, then $\operatorname{Ker}(W(R) \rightarrow W(K))$ is nil.

Proof. If $[E]$ lies in $\operatorname{Ker}(W(R) \rightarrow W(K))$, then for all maximal ideals $M$ of $R$, the class $\left[E \otimes_{R} R_{M}\right]$ lies in $\operatorname{Ker}\left(W\left(R_{M}\right) \rightarrow W(K)\right)$. By Theorem 2.4 then, $\left[E \otimes_{R} R_{M}\right.$ ] is nilpotent and so Corollary 2.7 completes the proof.

3. Miscellaneous results.

Proposition 3.1. Let $R$ be a regular noetherian domain. If all orderings of $K$ are archimedean, then $K(P)$ is not formally real for all nonzero prime ideals $P$ of $R$.

2 This is part of Theorem 1 of A.W. M. Dress, The weak local global principle in algebraic K-theory, to appear in Communications in Algebra. 
Proof. The proof is essentially the same as for the case of a Dedekind ring given in [9, Proposition 2.14(i)] and is reproduced here for the convenience of the reader. If $P \neq 0$ is a prime ideal of $R$ with $K(P)$ formally real, let $\tau$ be a signature of $K(P)$. The composition $U\left(R_{P}\right) \rightarrow U(K(P)) \stackrel{\tau}{\rightarrow}$ $\{ \pm 1\}$ then induces a signature $\sigma$ on the regular local ring $R_{P}$. By Corollary 2.5 (a), $\sigma$ extends to an ordering < of $K$. But then since $\sigma\left(1+P R_{P}\right)=1$, we have $1-x>0$ for all $x$ in $P R_{P}$, i.e., $x<1$ for all $x$ in $P R_{P}$. Such an ordering is clearly nonarchimedean, providing the desired contradiction.

Next, we give an example to show that if $R$ fails to be regular $\operatorname{Ker}(W(R) \rightarrow W(K))$ need not be nil.

Proposition 3.2. Let $R$ denote an integral domain such that $K$ is not formally real and possessing a maximal ideal $P$ with $K(P)$ formally real. Then, if $\phi: W(R) \rightarrow W(K)$ denotes the natural map, Ker $\phi$ is nonzero and not nil.

Proof. Since $K(P)$ is formally real the form $(1,1, \ldots, 1), r$ times, is anisotropic for all natural numbers $r>0$. Hence $r=r \cdot 1 \neq 0$ in $W(K(P))$ for all $r>0$. However, by [11, Satz 16] there is a natural number $m$ such that $2^{m}=0$ in $W(K)$. Hence $2^{m} \neq 0$ is a nonnilpotent element of Ker $\phi$.

Example. Let $R=\mathbf{R}\left[X_{1}, \ldots, X_{n}\right] /\left(X_{1}^{2}+\cdots+X_{n}^{2}\right), n \geq 5$. By [1, Exercise 5(b), p. 98], $R$ is a noetherian unique factorization domain, although it fails to be regular. Clearly, -1 is a sum of squares in $K$, so that $K$ is not formally real. If $P$ denotes the ideal generated by the images of the $X_{i}$ then $R / P \cong \mathbf{R}$. Hence $\operatorname{Ker}(W(R) \rightarrow W(K))$ is not nil. In addition to pointing out the necessity for regularity in Theorem 2.4 , this example also shows the need for regularity in Corollary 2.5 (b).

Remark. For nonintegrally closed domains it is easy to construct examples with $\operatorname{Ker} \phi \neq 0$, e.g., $R=\mathbf{Z}[3 i]$. However the above example shows that integral closure is not sufficient for the injectivity of $\phi$.

We have not been able to determine if $\operatorname{Ker} \phi=0$ in case $R$ is an arbitrary regular noetherian domain. However, in the case of polynomial rings over fields this is an easy consequence of a theorem of Karoubi [4]:

Theorem 3.3. Let $F$ be any field of characteristic $\neq 2$ and $X_{1}, \ldots, X_{n}$ indeterminates over $F$. Then $W\left(F\left[X_{1}, \ldots, X_{n}\right]\right) \stackrel{\phi}{\rightarrow} W\left(F\left(X_{1}, \ldots, X_{n}\right)\right)$ is injective.

Proof. It is well known that if $X$ is an indeterminate over $F$ then any anisotropic quadratic form over $F$ remains anisotropic over $F(X)$. Indeed, if $a_{1}, \ldots, a_{r}$ are nonzero elements of $F$ such that $\left(a_{1}, \ldots, a_{r}\right)$ becomes 
isotropic over $F(X)$ then, after clearing denominators, there will exist polynomials $P_{1}(X), \ldots, P_{r}(X)$ in $F[X]$, not all zero, such that $\sum_{i=1}^{r} a_{i} P_{i}(X)^{2}=0$. By dividing out the highest power of $X$ which divides all the $P_{i}(X)$ 's we can further assume that $P_{j}(0) \neq 0$ for some $j$. This yields an equation $\sum_{i=0}^{r} a_{i} P_{i}(0)^{2}=0$ with $P_{j}(0) \neq 0$ which shows that $\left(a_{1}, \ldots, a_{r}\right)$ is isotropic over $F$. By induction we conclude that, for indeterminates $X_{1}, \ldots, X_{n}$ over $F$, the natural map $\beta: W(F) \rightarrow W\left(F\left(X_{1}, \ldots, X_{n}\right)\right)$ is injective. By $[4$, Corollary 0.8 , p. 312 and Theorem 1.1, p. 317] the natural map $\alpha: W(F) \rightarrow$ $W\left(F\left[X_{1}, \ldots, X_{n}\right]\right)$ is an isomorphism. Since $\beta=\phi \circ \alpha$ it follows that $\phi$ is injective.

\section{BIBLIOGRAPHY}

1. N. Bourbaki, Éléments de mathématique. Fasc. XXXI. Chap. 7, Algèbre commutative, Actualités Sci. Indust., no. 1314, Hermann, Paris, 1965. MR 41 \#5339.

2. T. C. Craven, Witt rings and orderings of fields, $\mathrm{Ph}$. D. Thesis, Cornell University, Ithaca, N. Y., 1973.

3. I. Kaplansky, Rings and fields, Univ. of Chicago Press, Chicago, Ill., 1969. MR $42 \# 4345$.

4. M. Karoubi, Périodicité de la K-théorie hermitienne, Algebraic K-Theory III, Lecture Notes in Math., vol. 343, Springer-Verlag, Berlin and New York, 1973, pp. 301-411.

5. M. Knebusch, Grothendieck-und Wittringe von nichtausgearteten symmetrischen Bilinearformen, S.-B. Heidelberg. Akad. Wiss. Math.-Natur. Kl. 1969/70, 93-157. MR $42 \# 6001$.

6. - Specialization of quadratic and symmetric bilinear forms, and a norm theorem, Acta Arith. 24 (1973), 279-299. (to appear).

7. - Real closures of commutative rings. I, J. Reine Angew. Math.

8. M. Knebusch, A. Rosenberg and R. Ware, Structure of Witt rings and quotients of abelian group rings, Amer. J. Math. 94 (1972), 119-155. MR 45 \#5164.

9. - Signatures on semi-local rings, J. Algebra 26 (1973), 208-250.

10. D. G. Northcott, Ideal theory, Cambridge Tracts in Math. and Math. Phys., no. 42, Cambridge Univ. Press, Cambridge, 1953. MR 15, 390.

11. A. Pfister, Quadratische Formen in beliebigen Körpern, Invent. Math. 1 (1966), 116-132. MR $34 \# 169$.

12. O. Zariski and P. Samuel, Commutative algebra. Vol. II, University Series in Higher Math., Van Nostrand, Princeton, N. J., 1960. MR $22 \# 11006$. 96822

DEPARTMENT OF MATHEMATICS, UNIVERSITY OF HAWAII, HONOLULU, HAWAII 14853

DEPARTMENT OF MATHEMATICS, CORNELL UNIVERSITY, ITHACA, NEW YORK

DEPARTMENT OF MATHEMATICS, PENNSYLVANIA STATE UNIVERSITY, UNIVERSITY PARK, PENNSYLVANIA 16802 